\title{
Local Superfluidity at the Nanoscale
}

\author{
B. Kulchytskyy, ${ }^{1}$ G. Gervais, ${ }^{1}$ and A. Del Maestro ${ }^{2}$ \\ ${ }^{1}$ Department of Physics, McGill University, Montreal, H3A 2T8, Canada \\ ${ }^{2}$ Department of Physics, University of Vermont, Burlington, VT 05405, USA
}

(Dated: July 31, 2021)

\begin{abstract}
Motivated by the search for an experimentally realizable high density and strongly interacting one dimensional quantum liquid, we have performed quantum Monte Carlo simulations of bosonic helium-4 confined inside a nanopore with cylindrical symmetry. By implementing two numerical estimators of superfluidity corresponding to capillary flow and the rotating bucket experiment, we have simultaneously measured the finite size and temperature superfluid response of ${ }^{4} \mathrm{He}$ to the longitudinal and rotational motion of the walls of a nanopore. Within the two-fluid model, the portion of the normal liquid dragged along with the boundaries is dependent on the type of motion, and the resulting anisotropic superfluid density plateaus far below unity at $T=0.5 \mathrm{~K}$. The origin of the saturation is uncovered by computing the spatial distribution of superfluidity, with only the core of the nanopore exhibiting any evidence of phase coherence. The superfluid core displays scaling behavior consistent with Luttinger liquid theory, thereby providing an experimental test for the emergence of a one dimensional quantum liquid.
\end{abstract}

PACS numbers: 67.25.dr, 02.70.Ss, 05.30.Jp, 67.25.dj

\section{INTRODUCTION}

Superfluidity or dissipation-free flow, is rooted in quantum mechanics with the wave function of the entire fluid being described by an emergent global macroscopic phase $\theta$. In bulk ${ }^{4} \mathrm{He}$, this breaking of gauge symmetry has dramatic consequences for the liquid below the superfluid transition, $T_{\lambda} \simeq 2.17 \mathrm{~K}$. It is well established that superfluid helium can flow through extremely narrow constrictions, impenetrable to the normal liquid, with a velocity $\boldsymbol{v}_{s}=(\hbar / m) \boldsymbol{\nabla} \theta$ limited only by a critical velocity first understood by Landau. In "rotating bucket" experiments, where a container of superfluid is rotated at an angular frequency $\omega$, vortices can be spontaneously created, yielding a non-zero quantum of fluid circulation $\kappa=\oint \boldsymbol{v}_{s} \cdot d \boldsymbol{r}=\frac{h}{m} W$, where $W \in \mathbb{Z}$ is the topological winding number, equal to the number of vortices within a closed circulation loop. The quantitative details of the superfluid state were first probed in the celebrated Andronikashvili torsional oscillator experiment in 1946 where it was determined that a superfluid fraction of the total fluid does not contribute to the classical moment of inertia. This observation led to the development of Tisza's phenomenological two-fluid model where the superfluid state is understood as two intertwined liquids, having normal $\left(\rho_{n}\right)$ and superfluid $\left(\rho_{s}\right)$ components with total density $\rho=\rho_{n}+\rho_{s}$.

The superfluid-normal transition in bulk ${ }^{4} \mathrm{He}$ is in the three dimensional (3d) XY universality class. As the spatial dimension of the system is reduced, the enhancement of fluctuations suppresses the transition temperature to zero in $2 d$ and precludes any long range ordered state in one dimension. It is intriguing to consider how the continuum two-fluid picture holds up in the low dimensional limit where the bosonic helium system should be described by the universal harmonic Luttinger liquid (LL) theory ${ }^{11}$ at low energies. Such a correlated liquid is strongly fluctuating with any phase coherence decaying algebraically as a function of distance at $T=0 \mathrm{~K}$. Experimental realizations of low dimensional bosonic systems have been achieved in ultra-cold atomic gases ${ }^{22}$ at low densities where the interactions are expected to be weak and short ranged. At higher densities, and in the presence of strong interactions and Galilean invariance, direct observations of LL behavior are still lacking. Previous experimental work in low- $d$ quantum fluids has focused on superfluid helium confined to porous materials with a radial length scale in the nanometer range, and there is evidence for new quantum phases occurring at low temperatur $\mathrm{C}^{3 / 4}$ where the dynamical response can be understood in terms of LL theory $\sqrt{516}$. Recently, experiments have demonstrated the feasibility of measuring the superflow of helium through nanometer sized holes ${ }^{7}$. The next step will be to systematically decrease the radius of the nanopore, thereby providing a quasi- $1 d$ flow geometry in which the superfluid properties of helium can be measured. Unequivocal evidence of LL behavior in ${ }^{4}$ He filled nanopores will require a detailed understanding of the signatures of low dimensional superfluidity in the crossover regime, where fluctuations and strong interactions compete with the effects of confinement. Towards this goal, we have performed large scale numerical simulations measuring the superfluid response of a strongly interacting confined quantum fluid of helium- 4 at high density. The results expose a breakdown of the two-fluid model of superfluidity at the nanoscale and provide constraints on the experimental parameters needed to observe an emergent $1 d$ quantum liquid far from the previously observed Tonks-Giradeau regime $e^{819}$.

We begin by defining a model Hamiltonian that describes ${ }^{4} \mathrm{He}$ confined inside nanopores and provide some details of our quantum Monte Carlo (QMC) method. After a careful description of how superfluidity can be measured via the linear response of the fluid to bound- 
ary motion, we present its temperature dependence below the bulk superfluid transition. An investigation of its nanoscale properties identifies the presence of anisotropic superfluidity originating from only the Luttinger liquid core region of pores with nanometer radii.

\section{CONFINED HELIUM-4}

The starting point is a system of helium- 4 confined inside a nanopore of radius $R$ and length $L$ formed as a cylindrical cavity in a slab of amorphous silicon nitride. The interactions between helium atoms, $U$ are modeled via the Aziz potentia ${ }^{\sqrt{10}}$ while confinement is achieved by combining the effects of short range repulsion with the walls of the pore and a long range dispersion force between helium and the surrounding medium. The result is a surface wetting potential, $V$, with a deep attractive minimum near the pore wall ${ }^{11}$ where the Lennard-Jones parameters have been chosen for $\mathrm{Si}_{3} \mathrm{~N}_{4}(\varepsilon=10.22 \mathrm{~K}$, $\sigma=2.628 \AA)$ to coincide with the nanofluidics experiments described above. The resulting quantum manybody Hamiltonian:

$$
\hat{H}=\sum_{i=1}^{N}\left[-\frac{\hbar^{2}}{2 m} \hat{\nabla}_{i}^{2}+\hat{V}\left(\boldsymbol{r}_{\boldsymbol{i}}\right)\right]+\sum_{i<j} \hat{U}\left(\boldsymbol{r}_{i}-\boldsymbol{r}_{j}\right)
$$

where $N$ is the number of atoms with mass $m$, can be exactly simulated using continuous space Worm Algorithm (WA) quantum Monte Carlo ${ }^{12}$. Within the path integral formulation, this method exploits the quantum-classical isomorphism, performing Metropolis sampling of $(d+1)$ dimensional configurations of bosons that can be visualized as worldlines or trajectories in an imaginary time $(\tau)$ direction 13 . For helium at finite temperature, the worldlines obey a periodicity condition in the additional dimension modulo identical particle permutations, owing to their bosonic symmetry. The superfluid response of the low temperature system is directly linked to the existence and properties of long connected worldline exchange cycles consisting of many individual atoms.

Our simulations employ a fixed chemical potential $\mu / k_{\mathrm{B}}=-7.2 \mathrm{~K}$ to ensure helium atoms in pores of $L=75 \AA$ and $R=3.0-15.0 \AA$ are in thermal contact with a bath held at saturated vapor pressure for temperatures between $0.5-2.25 \mathrm{~K}$.

\section{SUPERFLUID DENSITY}

In QMC, the superfluid density is measured using linear response theory by considering the effects of boundary motion 14 . Within the two-fluid model, it is supposed that a superfluid fraction $\rho_{s} / \rho$ will remain stationary while the normal portion $\rho_{n} / \rho$ will be dragged along with the walls of the container. In the nanopore geometry, two types of motion, depicted in the first row of Fig. 1 are possible: longitudinal motion of the walls along the

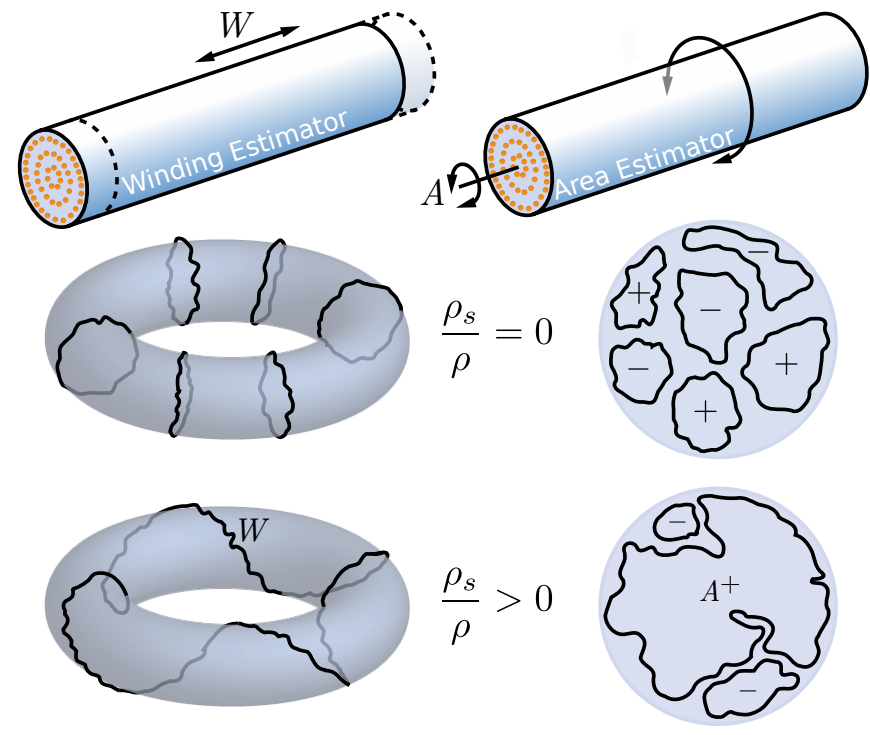

FIG. 1. (color online). The origin of superfluidity in ${ }^{4} \mathrm{He}$ filled nanopores measured by the winding (W, left column) and area (A, right column) estimators in the path integral representation. At high temperature, helium worldlines are short, containing only single particles and there is no superfluid fraction: $\rho_{s} / \rho=0$. As the temperature is lowered, the worldlines may link with each other, winding around periodic boundary conditions in the simulation cell and having a large projected net area perpendicular to the axis of rotation.

cylindrical axis and a rotation around it. These two types of response correspond to different measurements in the QMC, related to the geometry and topology of particle worldlines, represented as closed loops due to periodicity in imaginary time. Within the naive two-fluid picture, they should yield identical superfluid fractions and we can probe this notion by concurrently measuring the dynamical response to both types of boundary motion in the same nanopore.

\section{A. Longitudinal Response}

In the case of longitudinal wall motion, (left column, Fig. 11 we consider a container with periodic boundary conditions along the axis of the pore, and for the purposes of visualization, imagine the volume inside the pore to be mapped onto the surface of a torus. The major circumference of the torus is equal to $L$ while the minor one is $\hbar \beta$ with $\beta=1 / k_{\mathrm{B}} T$. In the path integral representation, the winding number is given by:

$$
W=\frac{1}{L} \sum_{i=1}^{N} \int_{0}^{\hbar \beta} d \tau\left[\frac{d z_{i}(\tau)}{d \tau}\right]
$$

where $z_{i}(\tau)$ is the $z$-component of $\boldsymbol{r}_{i}(\tau)$, the $(d+1)$ dimensional position of particle $i$. It is equivalent to the number of times the imaginary time trajectories of the $N$ 
particles wrap around the periodic boundary conditions of the sample. In WA simulations, the imaginary time $\tau$ must be discretized and we use $k_{\mathrm{B}} \Delta \tau / \hbar=0.004 \mathrm{~K}^{-1}$ to minimize Trotter error. The resulting superfluid density $\rho_{s}^{\mathrm{W}}$ is related to the variance of the distribution of winding numbers present $\frac{13 \mid 14}{1}$ through a winding estimator,

$$
\rho_{s}^{\mathrm{W}}=\frac{m L}{\pi R^{2} \hbar^{2} \beta}\left\langle W^{2}\right\rangle
$$

where $m$ is the mass of a helium atom and $\langle\cdots\rangle$ indicates a QMC average. At high temperature, the helium atoms behave classically, with spatially localized worldlines containing only a single atom. Long exchange cycles are extremely unlikely and so $\left\langle W^{2}\right\rangle=0$. As the temperature is lowered, the kinetic energy can be reduced by linking worldlines together. Such particle exchanges can be efficiently sampled within the WA using spatially local updates, producing configurations with extended worldlines that wind around the periodic boundary conditions $\left(\left\langle W^{2}\right\rangle \neq 0\right)$, producing a finite superfluid response.

\section{B. Rotational Response}

An alternative approach, equivalent in the $d \geq 3$ thermodynamic limit 15 , measures the non-classical response of the fluid to a small rotation. The superfluid fraction $\rho_{s}^{\mathrm{A}} / \rho$ is then equal to the non-classical rotational moment of inertia fraction $\left(I_{\mathrm{cl}}-I\right) / I_{\mathrm{cl}}$ where $I$ is the observed moment of inertia and $I_{\mathrm{cl}}$ is the total classical moment of inertia. The superfluid fraction defined in this way can be estimated in the QMC by measuring the worldline area $A$ of closed particle trajectories projected onto a plane perpendicular to the axis of rotation ${ }^{16}$ through an area estimator,

$$
\rho_{s}^{\mathrm{A}}=\frac{4 \rho m^{2}}{\hbar^{2} \beta I_{\mathrm{cl}}}\left\langle A^{2}\right\rangle
$$

For a rotation about the $z$-axis the path area is given by:

$$
A=\frac{1}{2} \sum_{i=1}^{N} \int_{0}^{\hbar \beta} d \tau\left[\boldsymbol{r}_{i}(\tau) \times \frac{d \boldsymbol{r}_{i}(\tau)}{d \tau}\right]_{z} .
$$

At high temperature, the projected mean squared areas are uncorrelated and $\sqrt{\left\langle A^{2}\right\rangle} \sim \Lambda^{2}$ where $\Lambda=$ $\sqrt{2 \pi \hbar^{2} \beta / m}$ is the thermal de Broglie wavelength and $\rho_{s}^{\mathrm{A}} / \rho \approx 0$ for large pores as seen in the right column of Fig. 1 As the temperature is reduced and long exchange cycles become energetically favorable, there is a distribution of finite projected areas and $\rho_{s}^{\mathrm{A}} / \rho>0$. The caveat to this approach is that angular momentum conservation requires that the dimensions of the simulation cell perpendicular to the axis of rotation do not have periodic boundary conditions. A quantum fluid confined inside a nanopore thus provides an ideal geometry where we can directly compare the two estimators of the superfluid fraction as shown in Fig. 2. The symbols correspond to QMC measurements performed using Eqs. (3) and (4) and the insets in the upper right corners are instantaneous particle configurations projected on the plane $z=0$. The solid line is the experimentally measured superfluid fraction of ${ }^{4} \mathrm{He}$ at saturated vapor pressure taken from Brooks and Donnelly $y \frac{17}{17}$ for comparison. For pores with $R<9 \AA$ we do not observe any superfluid response above $T=0.5 \mathrm{~K}$ while for $R \geq 9 \AA, \rho_{s}^{\mathrm{W}, \mathrm{A}} / \rho$ becomes non-zero at a $R$-dependent onset temperature shifted below the bulk value of $T_{\lambda}$. This is the expected behavior for a quantum fluid constrained inside a porous material where both $T_{c}$ and $\rho_{s} / \rho$ are reduced ${ }^{18 \mid 19}$ (see Ref. [20] for a review).

The observed superfluid response measured via the winding number is effectively one dimensional, originating from flow along the pore axis, and any non-zero value should be considered a finite size effect that will disappear as $L \rightarrow \infty$. In this limit, superfluidity can arise from the dynamical suppression of phase slips not captured in linear response theory ${ }^{\sqrt{5}}$. Another feature distinguishing the nanopore superfluid fraction from that of bulk helium is an apparent saturation at low temperature for both $\rho_{s}^{\mathrm{W}}$ and $\rho_{s}^{\mathrm{A}}$ at a value much less than one. A hint at the origin of this behavior can be observed by examining the spatial configurations inside the pore in Fig. 2. The interplay of interactions between helium atoms as well as with the surrounding amorphous $\mathrm{Si}_{3} \mathrm{~N}_{4}$ leads to states exhibiting a series of cylindrical shells $\mathrm{s}^{2122}$, equivalent to the formation of thin film layers of helium observed on $2 d$ substrates including silicate ${ }^{23}$.

\section{Local Superfluidity}

The competition between the tendency of bosons to delocalize at low $T$ and the strong geometrical confinement effects in the pore can be investigated by measuring the local contribution of the superfluid density. This was achieved by histogramming the radial $r$-dependence of the winding number ${ }^{24}$ or path area ${ }^{25}$

$$
\begin{aligned}
\rho_{s}^{\mathrm{W}}(r) & =\frac{m L^{2}}{\hbar^{2} \beta}\langle W \cdot W(r)\rangle \\
\rho_{s}^{\mathrm{A}}(r) & =\frac{4 m^{2}}{\hbar^{2} \beta I_{\mathrm{cl}}(r)}\langle A \cdot A(r)\rangle
\end{aligned}
$$

with $W$ and $A$ the full pore values defined above while

$$
W(r)=\frac{1}{2 \pi r L^{2}} \sum_{i=1}^{N} \int_{0}^{\hbar \beta} d \tau\left[\frac{d z_{i}(\tau)}{d \tau}\right] \delta\left(r-r_{i}^{\perp}(\tau)\right)
$$

and

$$
A(r)=\frac{1}{4 \pi r L} \sum_{i=1}^{N} \int_{0}^{\hbar \beta} d \tau\left[\boldsymbol{r}_{i}(\tau) \times \frac{d \boldsymbol{r}_{i}(\tau)}{d \tau}\right]_{z} \delta\left(r-r_{i}^{\perp}(\tau)\right)
$$

where $I_{\mathrm{cl}}(r)=m r^{2}$ is the classical moment inertia of a single helium atom and $r_{i}^{\perp}(\tau)=\sqrt{x_{i}^{2}(\tau)+y_{i}^{2}(\tau)}$. A 

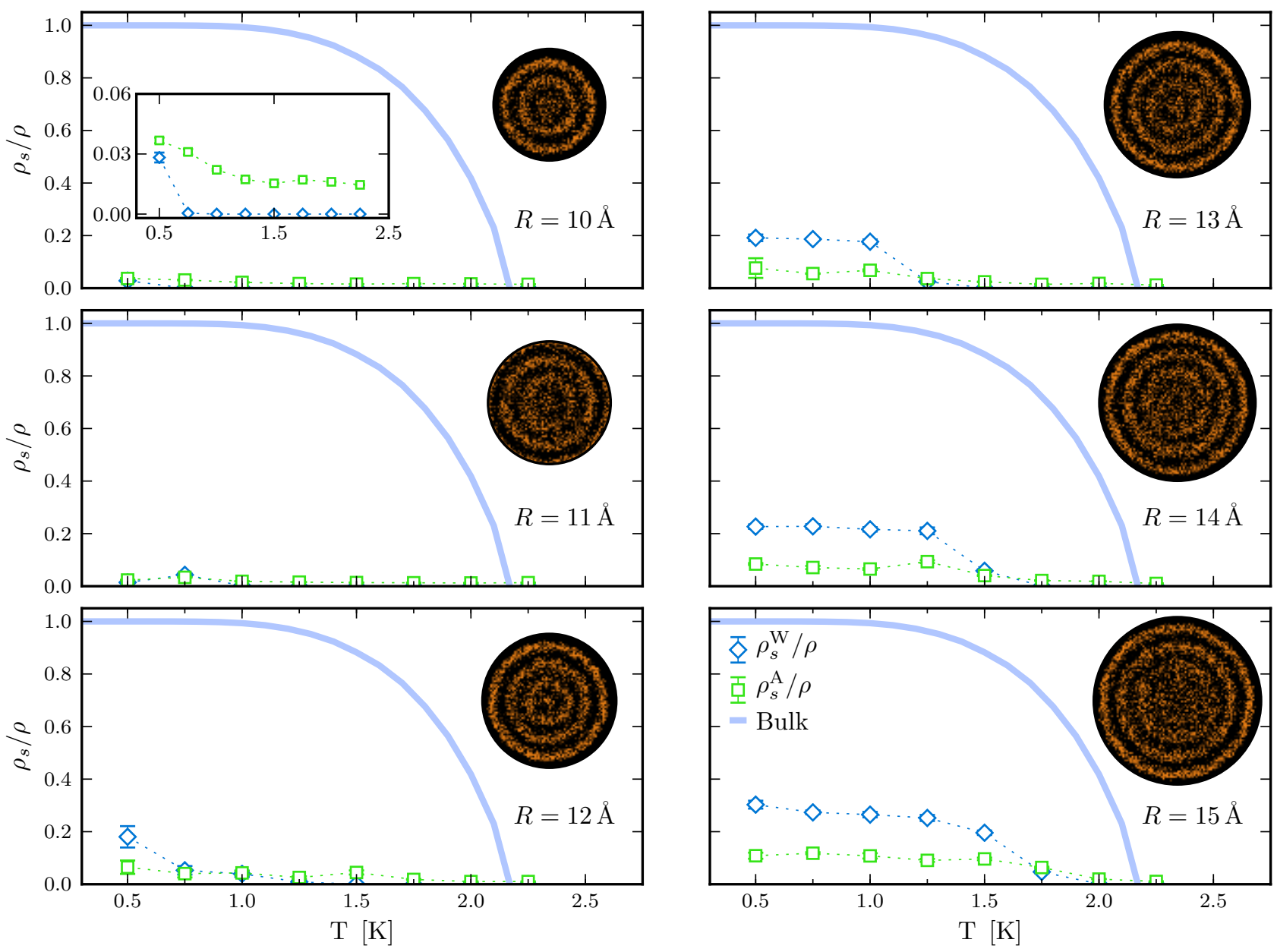

FIG. 2. (color online). A comparison of the superfluid fraction of helium confined inside nanopores with $L=75 \AA$ (symbols) measured using the winding number and projected area of particle worldlines with the experimentally measured value taken from Ref. 17] (line) for bulk ${ }^{4} \mathrm{He}$ at saturated vapor pressure. The upper right insets show instantaneous quantum Monte Carlo particle configurations projected onto the $z=0$ plane while the lower left inset in the first cell details superfluidity on a finer scale. All panels share the legend shown in the lower right panel.

comparison of the average particle number density with the two types of local superfluid density can be seen in Fig. 3 for a nanopore with $R=13 \AA$ and $L=75 \AA$ at $T=0.75 \mathrm{~K}$. The upper panels have been averaged over only the $z$-axis while the lower plot contains the fully cylindrically symmetric radial values defined in Eqs. (6) and (7). The saturation of the total superfluid density seen in Fig 2 can now be immediately understood in terms of a spatial "phase" separation where only the inner volume of the pore contains superfluid helium while the outer shells remain nearly solid, adhering to the walls. The results are qualitatively similar for $R=12-15 \AA$ with the two outermost shells making a negligible contribution to the superfluid density. The local superfluid estimators are nearly identical while their total values can differ by a factor of two. This presents no paradox due to their different normalizations:

$$
\begin{aligned}
\frac{\rho_{s}^{\mathrm{W}}}{\rho} & \equiv \frac{2 \pi L}{N} \int_{0}^{R} r d r \rho_{s}^{\mathrm{W}}(r), \\
\frac{\rho_{s}^{\mathrm{A}}}{\rho} & \equiv \frac{2 \pi L m}{I_{\mathrm{cl}}} \int_{0}^{R} r d r\left[r^{2} \rho_{s}^{\mathrm{A}}(r)\right]
\end{aligned}
$$

which are required to account for local contributions to the classical moment of inertia present in inhomogeneous fluids

$$
I_{\mathrm{cl}}=2 \pi L m \int_{0}^{R} r d r\left[r^{2} \rho(r)\right]
$$

and provide consistency with linear response theory 25 .

The temperature dependence of the local superfluid density can also be studied, and is shown for $R=13 \AA$ in Figure 4. When the total superfluid density exhibits 


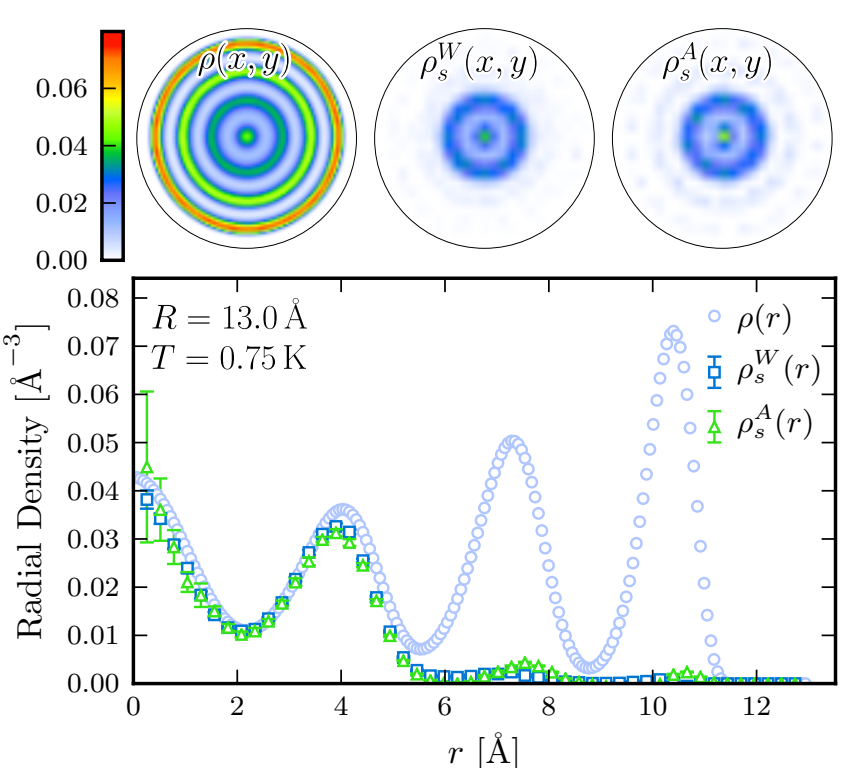

FIG. 3. (color online). The confined superfluid core seen by comparing the particle $(\rho)$, winding $\left(\rho_{s}^{\mathrm{W}}\right)$ and area $\left(\rho_{s}^{\mathrm{A}}\right)$ superfluid number densities measured via quantum Monte Carlo simulations for a $R=13 \AA$ radius nanopore of length $L=75 \AA$ at $T=0.75 \mathrm{~K}$. The first row shows the axially averaged local densities inside the pore while the lower plot contain an additional angular average.

a plateau, there is only a minimal dependence on temperature for the inner region of the pore. As the temperature is raised, the local superfluid density is reduced and begins to approach zero between the shells indicating a suppression of inter-shell particle exchanges. The non-zero and nearly temperature independent feature in $\rho_{s}^{A}(r)$ near the outer edge of the largest cylindrical shell appears for all radii studied, and is responsible for the finite value of $\rho_{s}^{A} / \rho$ at high temperature seen in the $R=10 \AA$ inset of Fig. 22. Its origin is connected to the presence of enhanced correlations in the worldline area when the radius of the cylindrical shell is on the order of the thermal de Broglie wavelength.

\section{LUTTINGER LIQUID CORE}

The core region exhibiting a non-zero superfluid response is nearly one dimensional, having a radius of $R \lesssim 6 \AA$. In $d=1$, fluctuations preclude the existence of any long range superfluid order, and instead, the helium system should be described at lowest order, by the linear quantum hydrodynamics of Luttinger liquid theory 1 with effective Hamiltonian

$$
H=\frac{\hbar v}{2 \pi} \int_{0}^{L} d z\left[\frac{1}{K}\left(\partial_{z} \phi\right)^{2}+K\left(\partial_{z} \theta\right)^{2}\right] .
$$

The phases $\phi(z)$ and $\theta(z)$ are defined in terms of the second quantized helium field operator $\psi^{\dagger}(z) \sim$
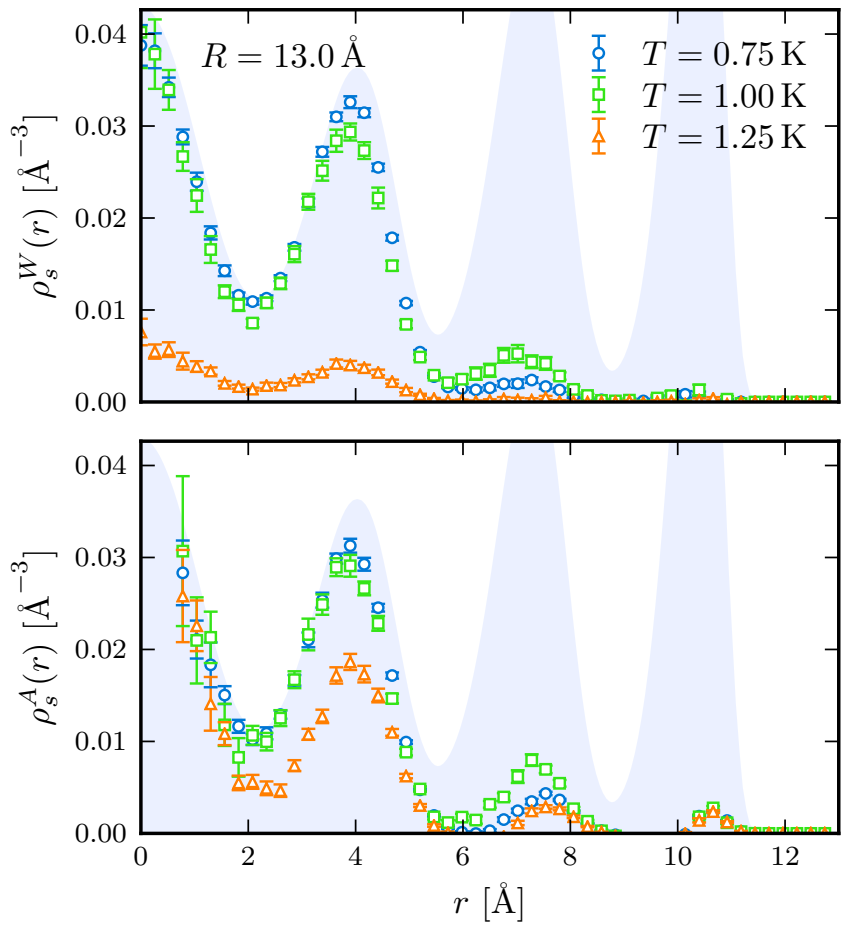

FIG. 4. (color online). The temperature dependence of the local superfluid density measured via the winding number (top) and area (bottom) estimator for a nanopore with radius $R=13.0 \AA$ and length $L=75 \AA$. The shaded region in the background corresponds to the particle density $\rho$ at $T=$ $0.75 \mathrm{~K}$.

$\sqrt{\partial_{z} \theta(z)} \mathrm{e}^{i \phi(z)}$ such that $\left[\phi(z), \partial_{z^{\prime}} \theta\left(z^{\prime}\right)\right]=i \pi \delta\left(z-z^{\prime}\right)$. Its low energy modes have dispersion $\varepsilon(k)=\hbar v k$ and the value of the Luttinger parameter $K$ tunes the system between algebraic superfluid $(K \ll 1)$ or solid $(K \gg 1)$ order. For a real physical system, the velocities $v_{J} \equiv v / K$ and $v_{N} \equiv v K$ can be related to the parameters of the underling many-body Hamiltonian. By comparing the predictions of harmonic LL theory, derived from the grand partition function $\mathcal{Z}=\operatorname{Tr} \exp [-\beta(H-\mu N)]$ with the measurements from finite temperature QMC simulations, $v_{J}$ and $v_{N}$ can be determined. For quasi- $1 d$ helium confined inside nanopores with $R<3 \AA$, this has already been accomplished ${ }^{26}$ but for larger radius pores, required the use of an ad hoc cutoff radius when analyzing QMC data. The physical origin of this cutoff is now fully understood as the radius of the superfluid core, and we expect it to be described by LL theory 27 :

$$
\frac{\rho_{s}^{W}}{\rho_{c}}=1-\frac{\pi \hbar \beta v_{J}}{L}\left|\frac{\theta_{3}^{\prime \prime}\left(0, \mathrm{e}^{-2 \pi \hbar \beta v_{J} / L}\right)}{\theta_{3}\left(0, \mathrm{e}^{-2 \pi \hbar \beta v_{J} / L}\right)}\right|
$$

where $\theta_{3}(z, q)$ is a Jacobi theta function with $\theta_{3}^{\prime \prime}(z, q) \equiv$ $\partial_{z}^{2} \theta_{3}(z, q)$ and $\rho_{c}=\left(N_{c} / N\right) \rho$ where $N_{c}$ is the number of atoms in the core. For each radius, we have performed a rescaling of the total superfluid response displayed in Fig. 2 and determined the velocity $v_{J}(R)$ through a fit- 


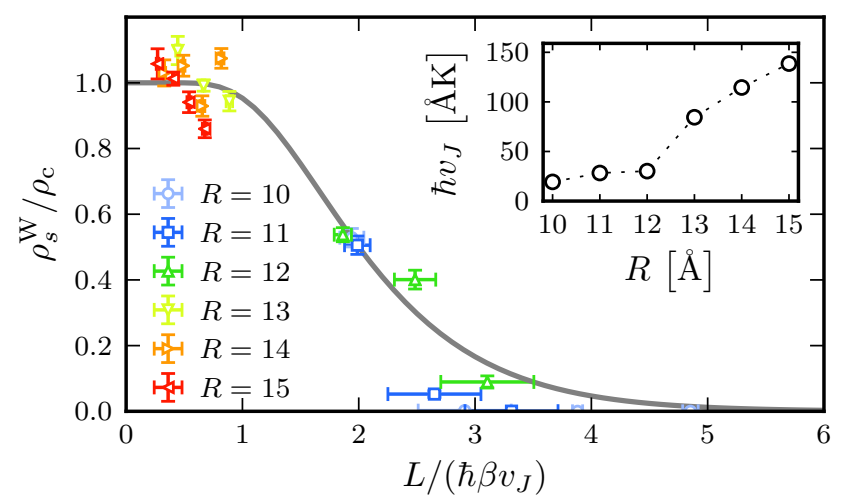

FIG. 5. (color online). The superfluid fraction of the core of nanopores for varying radii which can be collapsed onto the universal prediction from Luttinger liquid theory. The inset shows the extracted value of the phase velocity $\hbar v_{J}$ obtained by fitting to the winding number estimator for each radius.

ting procedure that yields the best collapse of all low temperature data onto Eq. (14). The results are displayed in Fig. 5 where the temperature scaling of the nanopore superfluidity is consistent with Luttinger liquid theory.

Much remains to be done, including confirming the predicted pore length scaling of $\rho_{s}^{\mathrm{W}} / \rho_{c}$ and evaluating the $R$-dependent LL parameter $K$. In addition, it seems natural to contemplate the effects of disorder, surely present in the pore walls, as well as the introduction of fermionic ${ }^{3} \mathrm{He}$ which may strongly alter superfluidity as bosonic exchanges will be suppressed in $1 d$.

\section{CONCLUSIONS}

We have performed large scale quantum Monte Carlo simulations for helium- 4 confined inside short $75 \AA$ pores with radii between $1-1.5 \mathrm{~nm}$. The results show a finite and anisotropic superfluid response above $T=0.5 \mathrm{~K}$, with a magnitude that is dependent on whether longitudinal or rotational motion of the nanopore is considered. The difference is large and arises from the absence of any classical moment of inertia in the truly $1 d$ limit where flow is still possible. Experiments probing this remarkable breakdown of the two-fluid picture could be performed by comparing the superfluid fraction measured by capillary flow and a nanoscale Andronikashvili torsional oscillator. Our results also indicate that when the radii of nanopores becomes sufficiently small, the superfluid fraction may exhibit plateaus, increasing in steps, due to the classical sticking of wetting layers near the pore walls. This is in stark contrast to the usual smooth temperature dependence of $\rho_{s} / \rho$ observed for bulk ${ }^{4} \mathrm{He}$ and could provide a signature of the crossover to $1 d$ behavior. If the fraction of atoms adhering to the nanopores walls could be discerned, possibly by comparing flow rates at high and low temperature, an examination of the finite size and temperature scaling of the superfluid density would confirm that confined low-dimensional helium is a Luttinger liquid. This would open up an exciting strongly interacting and high density regime where the effective low energy theory can be experimentally tested in systems with Galilean invariance.

We acknowledge financial support from the University of Vermont, NSERC (Canada), FQNRT (Québec), and the Canadian Institute for Advanced Research (CIFAR). This research has been enabled by the use of computational resources provided by the Vermont Advanced Computing Core supported by NASA (NNX-08AO96G), WestGrid, SHARCNET and Compute/Calcul Canada.
1 F. D. M. Haldane, Phys. Rev. Lett. 47, 1840 (1981)

2 M. Cazalilla, R. Citro, T. Giamarchi, E. Orignac, and M. Rigol, Rev. Mod. Phys. 83, 1405 (2011).

${ }^{3}$ K. Yamamoto, Y. Shibayama, and K. Shirahama, Phys. Rev. Lett. 100, 195301 (2008)

4 J. Taniguchi, R. Fujii, and M. Suzuki, Phys. Rev. B 84, 134511 (2011).

5 T. Eggel, M. A. Cazalilla, and M. Oshikawa, Phys. Rev. Lett. 107, 275302 (2011)

○ J. Taniguchi, K. Demura, and M. Suzuki, Phys Rev B 88, 014502 (2013).

7 M. Savard, G. Dauphinais, and G. Gervais, Phys. Rev. Lett. 107, 254501 (2011)

${ }^{8}$ T. Kinoshita, T. Wenger, and D. S. Weiss, Science 305, 1125 (2004).

9 B. Paredes, A. Widera, V. Murg, O. Mandel, S. Fölling, I. Cirac, G. V. Shlyapnikov, T. W. Hänsch, and I. Bloch,
Nature 429, 277 (2004).

10 R. A. Aziz, V. P. S. Nain, J. S. Carley, W. L. Taylor, and G. T. McConville, J. Chem. Phys. 70, 4330 (1979).

${ }^{11}$ G. J. Tjatjopoulos, D. L. Feke, and J. A. Mann, J. Phys. Chem. 92, 4006 (1988).

12 M. Boninsegni, N. V. Prokof'ev, and B. V. Svistunov, Phys. Rev. E 74, 036701 (2006).

13 D. M. Ceperley, Rev. Mod. Phys. 67, 279 (1995)

14 E. L. Pollock and D. M. Ceperley, Phys. Rev. B 36, 8343 (1987)

15 N. Prokof'ev and B. Svistunov, Phys. Rev. B 61, 11282 (2000)

10 P. Sindzingre, M. L. Klein, and D. M. Ceperley, Phys. Rev. Lett. 63, 1601 (1989)

17 J. S. Brooks and R. J. Donnelly, J. Phys. Chem. Ref. Data 6, 51 (1977).

18 J. Beamish, A. Hikata, L. Tell, and C. Elbaum, Phys. Rev. 
Lett. 50, 425 (1983)

${ }_{19}$ D. Finotello, K. A. Gillis, A. Wong, and M. W. H. Chan, Phys. Rev. Lett. 61, 1954 (1988).

20 J. D. Reppy, J. Low Temp. Phys. 87, 205 (1992)

21 E. S. Hernández, J. Low Temp. Phys. 162, 583 (2010).

${ }^{22}$ M. Rossi, D. E. Galli, and L. Reatto, Phys. Rev. B 72, 064516 (2005), J. Low Temp. Phys. 146, 95 (2006)

${ }_{23}$ M. Boninsegni, J. Low Temp. Phys. 159, 441 (2010).
24 S. A. Khairallah and D. M. Ceperley, Phys. Rev. Lett. 95, $185301(2005)$.

25 Y. Kwon, F. Paesani, and K. B. Whaley, Phys. Rev. B 74, $174522(2006)$

${ }_{20}$ A. Del Maestro, M. Boninsegni, and I. Affleck, Phys. Rev. Lett. 106, 105303 (2011)

27 A. Del Maestro and I. Affleck, Phys. Rev. B 82, 060515(R) (2010) 